\title{
La política de parques y ciudades industriales en México: etapa experimental (1953-1970)
}

\section{Gustavo Garza*}

De 1953 a 1986 se han construido en México 130 parques y ciudades industriales que constituyen una de las más importantes acciones realizadas para promover la industrialización en zonas atrasadas e intentar prever la elevada concentración industrial en las ciudades de México, Monterrey y Guadalajara. El desarrollo de este sistema de parques y ciudades industriales se puede periodizar en dos grandes etapas: i) la etapa experimental, 1953-1970; y, ii) la etapa de expansión, 1971 1987. En este contexto, este artículo analiza las características generales de la etapa experimental de este sistema para evaluar sus resultados, en espera de analizar la etapa de expansión en la continuación del proyecto en que se inscribe este trabajo.

Mientras los países desarrollados enfrentan el reto de reindustrializarse, los subdesarrollados continúan esforzándose por alcanzar los niveles de industrialización inicial necesarios para superar sus ancestrales carencias materiales y culturales. El desarrollo económico relativo que algunos países del Tercer Mundo habían logrado en décadas pasadas, como es el caso de México, se ha visto alarmantemente interrumpido en los años ochenta. Ante la crisis económica generalizada, emergen como prioritarios los problemas de la deuda externa, la espiral inflacionaria, el desempleo, la contracción de los mercados internos, los déficits en la balanza comercial, la inestabilidad de los mercados financieros, etcétera. Los esfuerzos del Estado se vuelcan a enfrentar estos retos insoslayables y las políticas urbano-regionales tienden a desplazarse, inexorablemente, a un segundo plano.

En el caso mexicano el papel del Estado para fomentar la industrialización ha sido doble. En primer lugar, por medio de la inversión pública y el crecimiento de las empresas paraestatales: por ejemplo, la participación pública en la formación bruta de capital fijo del país se ha elevado de $26 \%$ en 1970 a $52 \%$ en 1985 (Nacional Financiera, 1986: 69-70). En segundo lugar, por una serie de acciones dentro de las políticas fiscales

* Profesor-investigador del Centro de Estudios Demográficos y de Desarrollo Urbano de El Colegio de México. Este artículo presenta avances del proyecto titulado "Planeación urbana y cambio tecnológico en México", que se realiza bajo mi coordinación en el Centro de Estudios Demográficos y de Desarrollo Urbano de El Colegio de México. La investigación se efectúa dentro del Programa sobre Ciencia, Tecnología y Desarrollo (Procientec) dirigido por Alejandro Nadal y bajo los auspicios financieros del International Development Research Center de Canadá a quienes manifiesto mi agradecimiento por el apoyo brindado. Igualmente extiendo mi gratitud a la Secretaría de Desarrollo Urbano y Ecología, que tuvo a bien financiar una segunda etapa del trabajo de campo del proyecto. 
y monetarias, tales como la creación, en 1934, de Nacional Financiera (que se constituyó en el principal banco de fomento a la inversión), la protección industrial por licencias de importación, exenciones fiscales a la exportación; control de precios, concesiones de depreciación acelerada, etc. En el presente gobierno de Miguel de la Madrid se ha considerado que este modelo de crecimiento condujo a la actual crisis económica y, además, se ha agotado. Para superar la crisis se intenta hacer competitiva la economía modernizando el aparato productivo al abrir la economía hacia el exterior y liberalizar los mercados internos.

Existe un conjunto de condiciones tecnológicas, económicas, políticas y sociales, apenas anunciadas por el colapso de la bolsa mexicana de valores de octubre de 1987, que impiden que esta estrategia pueda tener éxito en un plazo previsible. Tarde o temprano, tendrá que ser ajustada para adecuarla a las posibilidades reales de la nación y para salvaguardar la soberanía de ésta. Así, se puede decir que cualquier tipo de reorientación en la política económica se tendrá que enmarcar en un plan general de desarrollo que integre las dimensiones sectorial y espacial de la economía.

Las políticas monetarias, fiscales, de ciencia y tecnología, etc., necesarias para estimular al sector industrial, requerirán apoyarse en los factores geográficos y del sistema urbano que inciden directamente sobre el proceso industrial. La articulación de las políticas sectoriales con las variables espaciales permitirá diseñar una estrategia explícita de localización industrial que hasta ahora no ha sido posible estructurar.

En esta dirección, el propósito del presente artículo es analizar las características generales de la etapa experimental del programa mexicano de parques y ciudades industriales que va de 1953 a 1970 , y evaluar sus resultados. Se considera que estos instrumentos son la política más consistente en materia de descentralización y fomento industrial en México y que se pueden transformar para aglutinar las acciones espacio-sectoriales que intentan reconvertir el aparato industrial del país.

\section{Conceptos de parque y ciudad industrial}

Los parques y ciudades industriales tienen diferentes aspectos distintivos, y existen diversas definiciones de ellos que atienden a sus rasgos físicourbanísticos.

Las definiciones de parque industrial que enfatizan sus características físicas son las más conocidas: se considera al parque como una porción de tierra dotada de infraestructura y servicios básicos tales como electricidad, agua, comunicaciones etc., que cuenta con edificaciones disponibles para vender o rentar a empresas industriales. Especificamente, se tienen las siguientes concepciones: 
i) Un parque industrial, como conjunto de industrias planeadas, provee al empresario de una selección de terrenos dotados de diversas instalaciones y calles diseñadas para la construcción de plantas modernas en un suelo protègido de usos no deseables (Porter, s/f: 29).

ii) El parque industrial es un conjunto organizado de establecimientos industriales provisto de ciertos servicios comunes e instalaciones construidas con antelación a la solicitud de compra y establecido como resultado de la iniciativa y planificación de una organización independiente (Bale, 1974: 291; y Matthews, cittado en Barr, 1983: 426).

iii) El parque industrial es un instrumento técnico que aglutina a un grupo planeado de empresas industriales ofreciendo lotes urbanizados, instalaciones y servicios. Es, a su vez, un proyecto organizativo de un grupo de fábricas construidas para proveer servicios comunes con el propósito de asistir y guiar a un amplio número de pequeños empresarios proporcionando incentivos a la expansión de la producción (Bharti, 1978: 7-10).

A partir de estas concepciones, se suelen incluir otros elementos que constituyen nuevas características del parque industrial. W. Bredo, autor de un estudio clásico sobre el tema, lo define de la siguiente forma:

\footnotetext{
"Un parque industrial es una superficie de tierra subdividida y urbanizada, según el plan comprensivo, para el uso de un conjunto de empresas industriales. El plan debe hacer una previsión detallada de calles y carreteras, transporte e instalación de infraestructura, así como construir edificios fabriles disponibles para su venta o renta. El plan debe asegurar un control adecuado del lugar y sus edificaciones a través de la zonificación, requerimientos legales en contratos de venta o renta y, por la provisión de una administración permanente, proteger la inversion del promotor del parque y de los compradores" (Bredo, 1960: 1).
}

La definición de Bredo agrega a la descripción física del parque un aspecto organizativo que se traduce en la existencia de una administración permanente encargada de su buen funcionamiento. Adicionalmente, las Naciones Unidas señalan en uno de sus estudios sobre el tema que los parques deben ; “...incluir ...un conjunto de servicios comunes, cuya variedad depende de las necesidades de las empresas y de la decisión de la administración del parque” (Naciones Unidas, 1968: 139).

Se puede concluir que el concepto de parque industrial se refiere simultáneamente a cuatro características: i) su objetivo básico de constituirse como espacio para el establecimiento de empresas industriales, construido con anticipación a su venta; ii) su aspecto físico-arquitectónico (superficie de tierra, edificios, calles, infraestructura); iii) los servicios comunes que proporciona (almacenes, edificios de administración, bancos, correos, dispensarios, escuelas de capacitación, transporte público, servicio médico, etc.); iv) su administración interna permanente. 
En síntesis, se puede definir al parque industrial como un instrumento constituido por una superficie de tierra diseñada para promover el establecimiento de empresas industriales, mediante la planeación y dotación anticipada de infraestructura, naves industriales y servicios comunes, todo lo cual debe funcionar bajo una administración institucional y permanente.

Las definiciones anteriores no incorporan otros elementos secundarios de los parques que pueden ser muy importantes para alcanzar los objetivos o propósitos que persiguen, tales como tipo de propiedad, tamaño, localización, etc. Adicionalmente, tienen cierto grado de inaprehensibilidad derivada del hecho de que conciben a estos instrumentos como estructuras estáticas, cuando en realidad presentan un compartimiento dinámico que los ha hecho transformarse de agrupaciones estrictamente secundarias a terciarias, cuaternarias (administración e informática) y, aún quinarias (prospección y planeación a largo plazo), como es el caso de la existencia de parques tecnológicos.

También debe mencionarse que existen algunos instrumentos afines o semejantes al concepto de parque industrial que es importante diferenciar: i) área industrial, ii) zona industrial, iii) complejo industrial y iv) ciudad industrial.

i) Área industrial. Es una porción de terreno subdividido en lotes para vender o rentar con el fin de establecer y construir empresas fabriles. Las naves industriales no se construyen con anterioridad, ni el área posee servicios y administración común. Muchos países utilizan este intrumento en vez de un parque industrial, aunque le llamen como a este último. Las Naciones Unidas consideran que esto es así porque las áreas industriales implican un reducido costo de inversión y presentan el incentivo de contar con cierto acomodamiento para que se establezcan industrias de todo tipo; en estas áreas pueden eventualmente desarrollarse parques industriales propiamente dichos. Los términos distrito industrial, subdivisión industrial, barrio industrial, son sinónimos de área industrial si se considera que se desarrollan en un medio ambiente no planeado y que utilizan instalaciones y edificios originalmente construidos para otros fines (Barr, 1983: 427-428).

ii) Zona industrial. Es sencillamente una superficie de tierra dentro o fuera de la ciudad que ha sido designada oficialmente para uso industrial. Puede no estar urbanizada pero, a diferencia del área industrial, presenta otros usos del suelo tales como residencial y comercial. La zona industrial es el término frecuentemente utilizado en la planeación urbana cuando, según ciertas características urbanísticas, geográficas y sociales, se establecen áreas de la ciudad para la localización de empresas industriales. Algunos autores señalan que la zona industrial debe considerarse como un lugar donde no se ha hecho ningún tipo de acondicionamiento y que únicamente ha sido reservado para uso industrial. 
iii) Complejo industrial. Es aquella organización industrial constituida por una combinación de empresas tecnológica y económicamente interconectadas que se localizan en una región para utilizar sus ventajas naturales y económicas (Stephen Rutt, 1986: 425). Cuando una empresa se constituye en el núcleo principal del complejo se le conoce también como enclave industrial y puede constituir un parque o área industrial, según tenga las características que identifican a cada uno de estos conceptos.

iv) Ciudad industrial. La ciudad industrial se diferencia sustancialmente del parque industrial y constituye una opción alternativa. Siendo resultado de acciones enmarcadas dentro de una planeación urbana global y consideradas como ambiciosos experimentos sociales, son difícilmente definibles de manera aceptable. En términos generales se puede decir que las ciudades nuevas (new towns), son localidades planeadas de forma integral con un origen identificable - fecha de inicio de construcción o ceremonia formal de fundación-, caracterizadas por una estructura urbana peculiar definida según usos del suelo, infraestructura, servicios, y fundadas para alcanzar objetivos y propósitos prestablecidos (véase Galantay, 1975; Clapp, 1971; Aldringe, 1979). Cuando están relacionadas con el desarrollo del sector secundario, a estas ciudades nuevas se les puede denominar ciudades industriales.

La ciudad industrial incluye una o varias superficies para industrias que pueden clasificarse como áreas o parques industriales según han sido anteriormente definidos, pero también como áreas habitacionales, comerciales, de servicios y amenidades urbanas. Si, además, se establece en una pequeña localidad aislada o aún en una superficie deshabitada, se habrán de construir escuelas, hospitales, restaurantes, bares, tiendas, espacios para actividades recreativas, etc., de tal suerte que la localidad se constituye, relativamente hablando, en autosuficiente y económicamente independiente constituyendo una ciudad nueva.

\section{Desarrollo del sistema de parques y ciudades industriales}

En la investigación de la que este artículo forma parte, se realizó un análisis de los instrumentos desarrollados por el Estado mexicano que se pueden clasificar como parques y ciudades nuevas según se definieron en el apartado anterior. Se diseñó un cuestionario que se recolectó en cada uno de ellos que en conjunto constituyen un sistema que representa una de las más importantes acciones realizadas en México para promover la industrialización en zonas atrasadas e intentar frenar la elevada concentración industrial en las ciudades de México, Monterrey y Guadalajara.

El sistema de parques y ciudades industriales se inicia en el pais en 1953 con la construcción de Ciudad Sahagún y para 1986 había 130 de 


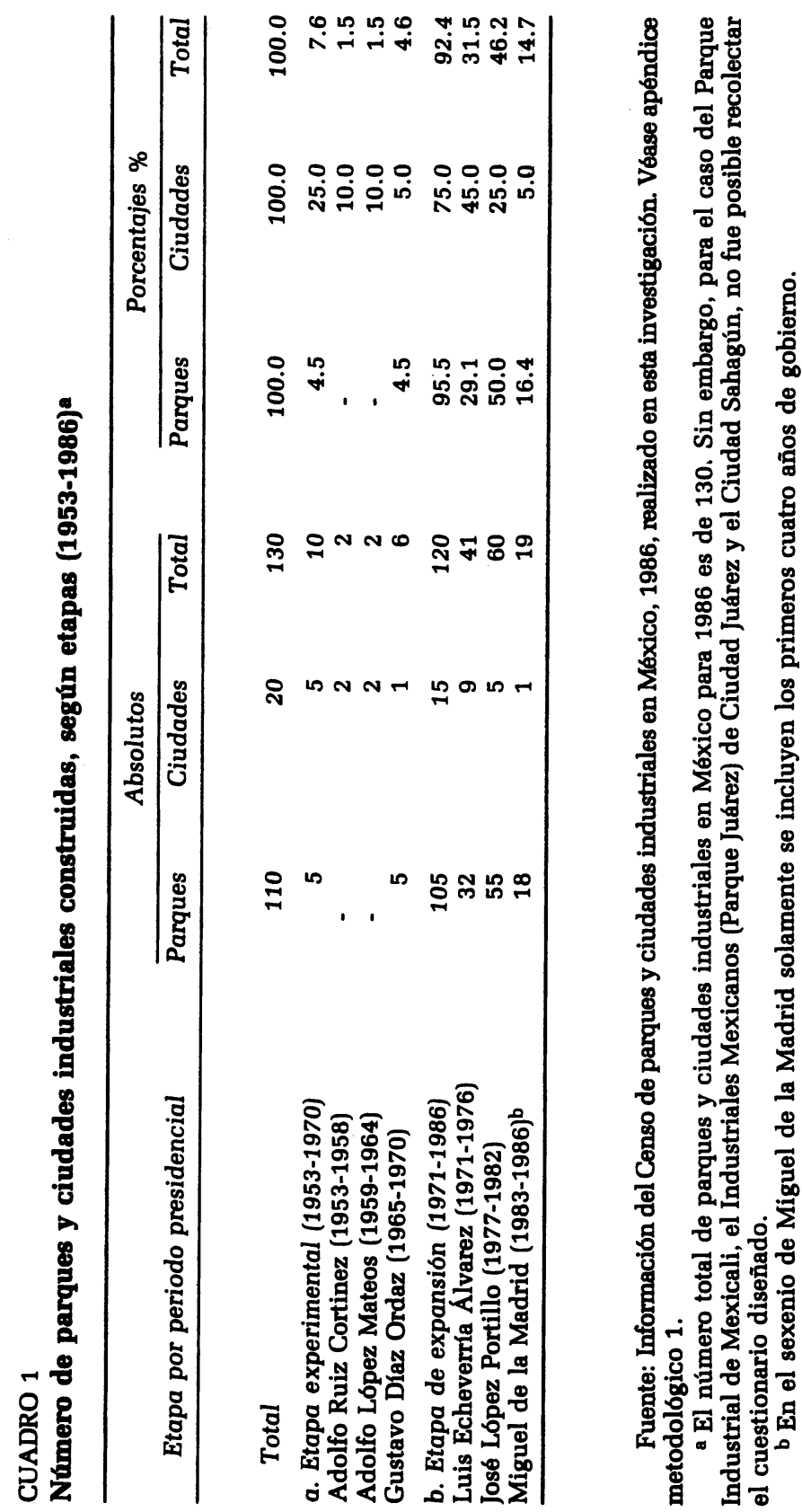


ellos. ${ }^{1}$ Su desarrollo se puede periodizar en dos grandes etapas que, a su vez, es posible dividir en periodos agrupados según los seis sexenios presidenciales transcurridos desde 1953: i) La etapa experimental, (19531970); es conveniente subdividirla en un periodo de parques estatales pioneros (sexenio de Adolfo Ruiz Cortines, 1953-1958), y en otro de lento despegue con la construcción de parques privados durante 1959-1970 y que cubre los sexenios de Adolfo López Mateos (1959-1964) y Gustavo Díaz Ordaz (1965-1970); ii) La etapa de expansión se inicia con el gobierno de Luis Echeverría Álvarez (1971-1976) y continúa con el de José López Portillo (1977-1982) y con los cuatro primeros años del actual gobierno de Miguel de la Madrid Hurtado (1983-1986). La primera etapa cubre 18 años y la segunda 16 y el criterio para delimitarlas fue el número de parques y ciudades industriales construidos, según se aprecia en el cuadro 1.

En la etapa que va de 1953 a 1970 únicamente se construyeron cinco ciudades y otros tantos parques, para hacer en total 10 de estos instrumentos, que representan únicamente $7.6 \%$ del sistema, por lo que se consideró justificado bautizarla como de carácter experimental. En 1971 se inicia una etapa de expansión con la construcción acelerada de parques y ciudades industriales, que hacia 1986 sumaban 120 para constituir, junto con los anteriores el actual sistema de 130 (véase el cuadro 1). Por razones de espacio sólo se analiza a continuación la etapa experimental, en espera de presentar en otra oportunidad el análisis del periodo de expansión.

\section{Etapa experimental de los parques y ciudades industriales (1953-1970)}

\section{a. Periodo 1953-1958: ciudades industriales pioneras}

El primer instrumento de descentralización industrial en México fue la Ciudad Bernardino Sahagún, construida en 1953. Fue diseñada en analogía a los new towns ingleses y planeada para albergar 60 mil habitantes. Su edificación se debe a que tres empresas paraestatales tomaron la iniciativa de localizarse fuera de la ciudad de México en correspondencia

\footnotetext{
${ }^{1}$ En esta investigación se detectó la existencia de 130 parques y ciudades industriales en toda la República pero, por diversas razones, en tres de ellos no se pudo recolectar el cuestionario diseñado: Parque Industrial Mexicali; Parques Industriales Mexicanos o Parque Juárez, en Cd. Juárez, y Fideicomiso Cd. Sahagún. Se puede mencionar la existencia de otros centros que tienen el nombre de parque pero son zonas industriales según se definieron en el inciso anterior: Parque Industrial Delta; y Parques Industriales de Nezahualcóyotl, Cuautitlán-lzcalli e Iztapalapa, los tres en el estado de México. En este artículo se consideraron los 130 parques, pero su análisis pormenorizado en la investigación general se hizo con base en los 127 cuestionarios recolectados.
} 
con el punto de vista del gobierno federal sobre la necesidad de revertir la tendencia hacia la elevada concentración industrial y demográfica en la ciudad de México. A principios de los años cincuenta la capital absorbía $40.4 \%$ del valor agregado bruto industrial (Garza, 1985: 143) y tenía 2.8 millones de habitantes, que representaban $11.1 \%$ de la población total nacional (véase Unikel, Ruiz, Garza, 1976: 134).

Para intentar neutralizar esta tendencia, con ciudad Sahagún se inicia el programa mexicano de parques y ciudades industriales. Su localización se determinó siguiendo dos criterios muy simples: en primer lugar, se consideró la dotación de infraestructura y algunos recursos naturales, especialmente agua. En el sitio seleccionado existía un gasoducto, líneas eléctricas de alta tensión y vías férreas; en segundo lugar, se pensó que sería una medida que contribuiría al desarrollo socioeconómico de Hidalgo, uno de los estados económicamente más atrasados de la República.

Ciudad Sahagún se localiza a $100 \mathrm{~km}$ de la ciudad de México, dentro de su región inmediata de influencia, por lo que en los inicios del programa se optó por una estrategia de descentralización intrarregional. De esta forma, se esperaba que las empresas que se establecieron en ella permanecerían cerca del principal mercado del país y de otros factores de localización que garantizarían su éxito.

Esto no ocurrió en Ciudad Sahagún, pues a pesar de haberse invertido 80 millones de dólares en la construcción de la infraestructura, en sus 34 años de existencia sólo una empresa se agregó a las tres fundadoras. ${ }^{2}$ A posteriori, parece fácil decir que su fracaso relativo se debe a su inadecuada localización. ${ }^{3}$ El área original no disponía de los factores locacionales requeridos para el funcionamiento de las empresas: trabajadores especializados, servicios bancarios y profesionales, disponibilidad de insumos, infraestructura desarrollada, mercado local, etc. Adicionalmente, la ausencia de "ambiente urbano" hizo difícil atraer empresarios y trabajadores acostumbrados a la vida metropolitana y sus consiguientes "amenidades". Sea como fuere, este new town mexicano fue el primer instrumento en el desarrollo de un programa cuya etapa experimental se analiza en este trabajo.

${ }^{2}$ Las empresas fundadoras fueron: Diesel Nacional, S.A,; Constructora Nacional de Carros de Ferrocarril, S.A.; Fábrica Nacional de Maquinaria Textil, y Toyoda de México, S.A. En la actualidad existen estas mismas empresas más Dina Komatsu, S. A.

${ }^{3}$ Se señala que Ciudad Sahagún está muy lejos de la ciudad de México para ser un suburbio y muy cerca para ser ciudad independiente. Sea como fuere, el nulo crecimiento de su base industrial hizo que "las grandes expectativas de economistas y planeadores mexicanos quienes construyeron la ciudad industrial han sido grandemente frustradas" (F.C. Miller, 1973: 100-102). Esto que se decía hace más de 13 años es aún más grave en la actualidad en el contexto de la crisis económica, pues Diesel Nacional está casi paralizada con $70 \%$ de su personal en "paro técnico" y no se considera remoto que se convierta en un pueblo fantasma (La Jornada, 1987, 27). 
En 1955 la Compañia Federal de Electricidad (CFE), asesorada por el gobierno federal, firma la escritura constitutiva de Ciudad Industrial Irapuato, que inicia sus operaciones en 1957 . Situada a $290 \mathrm{~km}$ de la capital del país y a $6 \mathrm{~km}$ de Irapuato, Guanajuato, localidad de $83 \mathrm{mil}$ habitantes especializada en empresas agroindustriales, esta ciudad no tuvo el éxito esperado. En 1960, tres años después del inicio de operaciones, “... un solo lote había sido vendido en todo el distrito industrial debido a que, en primer lugar, los empresarios lo consideraban muy lejano a la ciudad de Irapuato y, en segundo lugar, por el excesivo precio de la tierra" (Unikel, 1964: 4). Posteriormente, siete años después de su inauguración, 10 de los 90 lotes para industria ligera habían sido vendidos, pero sólo seis fueron ocupados.

En 1986, Ciudad Industrial Irapuato tiene 39 empresas cuyo tamaño de terreno varía desde los $3539 \mathrm{~m}^{2}$ hasta los $772120 \mathrm{~m}^{2}$ que tiene la dependencia encargada de la transmisión y distribución de energía eléctrica, la CFE. El promedio para las 39 empresas es de $33823 \mathrm{~m}^{2} \mathrm{y}$, descontando a la CFE, de $14402 \mathrm{~m}^{2}$. Treinta años después de haberse iniciado, aún están disponibles lotes en venta en dos secciones urbanizadas por un total de alrededor de $790 \mathrm{mil} \mathrm{m}^{2}$; esto es que, suponiendo $14 \mathrm{mil} \mathrm{m}^{2}$ en promedio por empresa, la ciudad podrá albergar 55 empresas más, que representan $140 \%$ de las que se establecieron eu 30 años. En este caso se evidencia, dramáticamente, la lenta rotación del capital de estas inversiones. Para asumirlas, se requiere el apoyo del Estado o, en su defecto, de una muy peculiar inversión privada que no requiera recuperar el capital en plàzos razonables por tener otras finalidades o estar integrada con otros proyectos.

Considerando que según estándares internacionales se requieren alrededor de 10 años para que un parque industrial se desarrolle completamente, el caso de Ciudad Industrial Irapuato está muy lejos de ser satisfactorio. Sin embargo, cabe destacar que su localización está claramente fuera de la región de influencia inmediata de la ciudad de México, por lo que se ajusta a un intento de descentralización interregional y que es una ciudad industrial tal como ha sido definida. Junto con Ciudad Sahagún, por tanto, conforman lo que hemos definido como ciudades industriales pioneras en México.

\section{b. Periodo 1959-1964: primera ciudad industrial privada}

Durante el sexenio de Adolfo López Mateos (1959-1964), se crearon dos ciudades industriales, una pública y otra privada, y el primer parque industrial privado en el país (véase el cuadro 1). Este último, denominado Parque Industrial Delta, lo inició en 1960 en León, Guanajuato, un fraccionador de la ciudad de México que después lo abandonó. Existe en la actualidad como zona industrial, por lo que se eliminó de esta investiga- 
ción. Cuenta con alumbrado público insuficiente, energía eléctrica, teléfono y agua domiciliaria, pero carece de pavimentación y drenaje pluvial. Las cuatro empresas que se localizan en esta zona realizan gestiones directamente con el ayuntamiento y no existe ningún tipo de administración interna, por lo que no es un parque en sentido estricto. ${ }^{4}$

El Parque Industrial Lagunero es una ciudad industrial según se ha definido. Se constituyó en 1962 y, al igual que la Ciudad Industrial de Irapuato, fue realizada por la CFE. Se localiza al oriente de la ciudad de Gómez Palacio, Durango, colindando con el cauce seco del Río Nazas, que lo separa del área urbana de Torreón, Coahuila. Estas dos ciudades, junto con la de Lerdo, Durango, constituyen una sola área metropolitana cuya localidad principal es Torreón, que se localiza a $987 \mathrm{~km}$ de la ciudad de México. El parque tiene una superficie total de 364 ha, de las cuales 234 son para uso industrial, 16 habitacional, 5 comercial, 93 de servicios comunes y 16 de áreas verdes y vialidad.

Antes de la creación de esta ciudad, la CFE había construido una planta eléctrica en esa zona y una de las razones para localizarla en ese lugar fue "crear un mercado para su creciente producción de energía eléctrica", (Unikel, 1964:4). En la actualidad, la ciudad industrial está totalmente construida, aunque se fue realizando durante un proceso de cinco etapas. Al principio se contaba con 120 lotes y las primeras empresas en instalarse fueron una planta de Petróleos Mexicanos, seis distribuidoras de productos de petróleo, una empresa de reparación de carros de ferrocarril y una agencia de automóviles.

Para las cinco etapas dispone en total de 422 lotes, divididos en 113 para pequeña industria ( $1700 \mathrm{~m}^{2}$ en promedio), 270 para mediana industria (8000 m en promedio) y 39 para gran industria $\left(25000 \mathrm{~m}^{2}\right.$ en promedio). Cada etapa que se ha construido ha sido totalmente urbanizada y actualmente cuenta con calles pavimentadas, aceras, agua, drenaje, energía eléctrica, alumbrado público, gasoducto, espuela de ferrocarril, etc. Su administración la realiza una entidad pública estatal denominada Promotora del Desarrollo Industrial y Urbano en la Región Lagunera de Durango (Prodinur). A 25 años de inicio, se han vendido todos los lotes y existen 360 empresas establecidas, siete en construcción y 10 programadas, mientras que los 45 lotes restantes están sin uso. Dado su gran número de empresas, se podría considerar como un proyecto exitoso, sin tomar en cuenta que no se han alcanzado los propósitos de descentralización industrial y reducción de las desigualdades regionales que persigue el sistema en su conjunto, a nivel nacional.

4 En la visita que se hizo a esta zona industrial se detectó la operación de 5 empresas industriales: Gamesa, S.A., Delso, S.A. de C.V. (fabricante de suelas de hule de calzado); Gonher, S.A. (fabricante de filtros automotrices); Pilsa, S.A. (productos plásticos); y Nibco de México, S.A. de C.V. (válvulas y conexiones metálicas). 
La Ciudad Industrial Valle de Oro, localizada en San Juan del Río, Querétaro, es el primer conjunto privado que se realiza en México. En 1963 se elabora su escritura constitutiva y dos años después se inicia su construcción. Tiene un tamaño de $\mathbf{3 0 0}$ ha y lotes para pequeña, mediana y gran empresa, que se subdividieron sobre un terreno que había sido expropiado por el gobierno del Estado y se vendió al promotor. A diferencia de las anteriores ciudades, que únicamente venden lotes urbanizados, ésta tiene lotes y naves industriales en renta. El propietario es el administrador que desempeña las labores de mantenimiento y atención de las firmas que solicitan rentar. En la actualidad, la ciudad cuenta con 120 empresas en operación y en sus casi 25 años de existencia se puede considerar que ha experimentado un desarrollo medianamente satisfactorio.

Así durante el gobierno de Adolfo López Mateos (1959-1964) se erigen únicamente una ciudad industrial pública y otra privada. Con esta última, se inicia la construcción privada de estos instrumentos que en el futuro serían parte importante del sistema de parques y ciudades industriales en México.

\section{c. Periodo 1965-1970: primer parque y fin de la etapa experimental}

Durante el sexenio de Gustavo Díaz Ordaz culmina la etapa experimental del sistema de parques y ciudades industriales en México. Se observa, en primer lugar, un aumento significativo en su número, pues se construyen seis nuevos proyectos, casi triplicando los cuatro existentes para totalizar 10 en 1970 (véase el cuadro 1). Cabría agregar que en estos años también surgen algunos 'centros industriales' que no constituyen parques o ciudades según las definiciones presentadas en el acápite anterior, tales como el Centro Industrial Barranquilla, de 1.1 ha y el Centro Industrial los Pinos de 4.4 ha, ambos localizados en Baja California Norte. Estos 'centros' son lotificaciones para uso industrial que conforman reducidas zonas industriales dentro de la ciudad.

En segundo lugar, destaca la construcción, por primera vez en México, de parques industriales estrictamente hablando - tal como se definieron en el inciso 1-; antes sólo existían cuatro ciudades industriales (véase el cuadro 1). Es importante hacer notar, sin embargo, que en este periodo la única diferencia entre ciudades y parques industriales consistió en el uso del suelo: era más diversificado en la primera (industrial, comercial, habitacional, de esparcimiento), que en el segundo (prácticamente sólo uso industrial). No hubo diferencias notables de tamaño o importancia económica y la mayor parte de los cinco parques del periodo son de mayor tamaño que, por ejemplo, la Ciudad Industrial del Valle de Cuernavaca (Civac). En general, no obstante, la ciudad industrial es un instrumento 
más complejo que el parque y requiere de mayores inversiones para su construcción. Que se hayan realizado cinco parques y sólo una ciudad industrial entre 1965 y 1970 revela que, por la carencia de financiamiento para construir ciudades industriales propiamente dichas, se optó por hacer parques por ser menos costosos al no contar con viviendas y otras edificaciones.

Sin embargo, si se considera el conjunto de parques y ciudades industriales del país, se comporta como se esperaría conceptualmente, pues mientras el tamaño promedio de los parques es de 119 ha, el de las ciudades es de 514 ha. Civac es, pues, una excepción, al ser la ciudad industrial más pequeña en México.

Finalmente, en tercer lugar, es significativo que cuatro de los cinco parques son privados y solamente uno público, pero no federal sino estatal, igual que la ciudad industrial, que fue una iniciativa del gobierno del estado.

El complejo proceso de trámites legales, construcción, promoción, ventas y obtención de financiamiento de ciertos parques industriales ameritaría un estudio de caso en profundidad, pero siendo el objetivo de la investigación en que se inscribe este artículo analizar todo el conjunto, esto no es posible. En lo que sigue se presentan esquemáticamente las características generales de los cinco parques y la ciudad industrial construidos entre 1965-1970, con el objeto de tener una idea del carácter del sistema en su conjunto.

En 1966 se firma la escritura constitutiva de la Ciudad Industrial del Valle de Cuernavaca (Civac), que en 1970 inicia su construcción sobre una superficie de 43 ha de terrenos ejidales que el gobierno del estado de Morelos había expropiado. El contrato del fideicomiso fue signado por el gobierno uel estado y el Banco Nacional de México (Banamex); éste se encargo de su promoción. Civac cuenta con alrededor de 100 lotes, de los cuales 25 son para pequeña industria (lotes promedio de $2200 \mathrm{~m}^{2}$ ), 60 para mediana (tamaño promedio de $8000 \mathrm{~m}^{2}$ ) y 15 para gran industria (de más de $10000 \mathrm{~m}^{2}$ de superficie). En la actualidad todos los lotes están vendidos, y cuando terminó la venta, Banamex dejó la empresa, estableciéndose la Administración de la Ciudad Industrial Valle de Cuernavaca (Procivac), encargada de su mantenimiento y administración, las cuales financia por cuotas de las empresas. Existen 88 firmas industriales establecidas, y en los lotes vendidos, pero desocupados, se encuentran en construcción siete más. El tamaño promedio de los lotes industriales es de $19200 \mathrm{~m}^{2}$ por empresa, pero existen algunas con únicamente $347 \mathrm{~m}^{2}$ y la mayor tiene $412297 \mathrm{~m}^{2}$.

Finalmente, la infraestructura de Civac es razonablemente adecuada para el funcionamiento de las empresas, a pesar de que el suministro de agua ha llegado al máximo nivel y no se permite establecer nuevas em- 
presas con elevado consumo del líquido. ${ }^{5}$ Desde el punto de vista de un proyecto individual podría considerarse como exitosa, puesto que a pesar de que requirió de $\mathbf{2 0}$ años para su completa realización, ha logrado atraer las empresas suficientes para cubrir su superficie disponible. A nivel macrorregional, su localización (a menos de 100 kilómetros del área metropolitana de la ciudad de México y dentro de su sistema de ciudades), puede considerarse errónea desde la perspectiva de una política nacional de descentralización industrial, pues ha contribuido al crecimiento acelerado de Cuernavaca, que en el futuro próximo se incorporará como pequeña zona metropolitana a la megalópolis de la ciudad de México (véase Garza, 1987: 419)

El Parque Industrial El Vigía, localizado en Mexicali, Baja California Norte, cuya acta constitutiva data de 1966, es el primer parque industrial propiamente dicho que se construyó en México. Su realización fue a iniciativa de un grupo de empresarios locales, por lo que es de carácter privado. Ha tenido un desarrollo muy accidentado y cuenta con 60 lotes distribuidos en 23 ha ( 30 de $1500 \mathrm{~m}^{2} ; 22$ de $1800 \mathrm{~m}^{2}$ y $18 \mathrm{de} 2000 \mathrm{~m}^{2}$ ), pero sólo tiene en la actualidad nueve empresas en operación después de 20 años de fundado. Sus propietarios, agricultores y empresarios de la industria de la construcción, concibieron el proyecto para construir naves industriales para rentar a empresas extranjeras dedicadas a la maquila. Construyeron inicialmente tres naves y no fue sino hasta en 1976 cuando se hicieron seis naves adicionales. Estas son rentadas por las nueve empresas establecidas a razón de 35 centavos de dólar el metro cuadrado en las nuevas y 25 en las antiguas. Los propietarios no han puesto en venta lotes ni naves industriales y sólo $15 \%$ del parque está ocupado. Por tanto, difícilmente podría calificarse de exitosa esta extraña iniciativa empresarial y cabría pensar que se ha seguido más un criterio de 'rentista agropecuario' que el de una moderna empresa inmobiliaria cuya minimización del tiempo de venta es crucial para la rentabilidad del proyecto. El auge de la industria maquiladora a partir de 1982 y la virtual saturación del Parque Industrial Mexicali (fundado en 1979, por lo que no se incluye en este trabajo), hacen pensar que estos pacientes empresarios tendrán más éxito en los años venideros.

En 1967 se firma el acta constitutiva de un segundo parque privado, el Parque Industrial Antonio J. Bermúdez, en Ciudad Juárez, Chihuahua. Contando con un total de 142 ha, es relativamente grande, pues tiene mayor tamaño que casi $\mathbf{8 0} \%$ de los 110 parques existentes. La organización del

\footnotetext{
5 En la actualidad, no obstante, la infraestructura tiende a deteriorarse y a saturarse por lo que "requiere poco más de 2000 millones de pesos para mejorar su infraestructura hidraúlica, pavimentar calles e instalar tanques de almacenamiento" (Excélsior, 5-11-87: 2). Esto ejemplifica el problema general de parques y ciudades industriales de no planear financieramente la obsolescencia y reemplazo de la infraestructura.
} 
parque contempla tanto la venta de lotes - se han vendido 26 - como su renta y la de naves industriales. En la actualidad se encuentran en operación 46 empresas, tres están en construcción y 10 existen programadas.

El tamaño de las empresas instaladas es considerable y sobresalen con mucho las que arman productos electrónicos. La superficie media de sus lotes es de $23390 \mathrm{~m}^{2}$, existiendo algunas muy grandes como la RCA Components, que tiene $93915 \mathrm{~m}^{2}$ de terreno y $30600 \mathrm{~m}^{2}$ de construcción, y otras pequeñas como Subensambles Electrónicos, con $3204 \mathrm{~m}^{2}$ de terreno y $7329 \mathrm{~m}^{2}$ de construcción. La infraestructura del parque es notoriamente superior a la de las zonas industriales en Ciudad Juárez, y está bien planeado y administrado. Específicamente, cuenta con calles y banquetas pavimentadas, red hidraúlica, drenaje pluvial, sanitario, líneas telefónicas, alumbrado público, así como redes de hidrantes y equipo contra incendio. La administración interna pertenece a un consorcio de empresas del dueño del parque y está dividida en varios sectores: comercial, promoción y construcción; conservación y mantenimiento y contabilidad y manejo financiero. A pesar de ser uno de los parques más caros de la ciudad y del país, la eficiencia en organización y éxito en promoción y ventas lo hacen parecer una inversión productiva, aunque de lenta rotación -cumple 20 años de existencia. El auge maquilador y su experiencia en la construcción y administración de este tipo de instrumentos, ha estimulado a sus propietarios a construir nuevos parques en Ciudad Juárez, así como a asesorar a otros promotores en varias partes de la República.

Incuestionablemente, este parque privado se puede considerar como una venturosa empresa comercial con resultados positivos para el desarrollo industrial de la ciudad. No obstante, lo que interesa estudiar es la estrategia global de descentralización industrial en México. Esto sólo se podrá hacer con el análisis agregado de todos los parques y ciudades industriales del país, que se realizará en las partes subsiguientes de la investigación en que se inscribe este artículo.

Los dos últimos parques industriales privados del periodo 1965-1970 fueron el Parque Industrial de Nogales (1968), situado en dicha ciudad del estado de Sonora en la frontera norte de México, y el Parque Industrial Cartagena (1970), localizado en el municipio de Tultitlán, estado de México, que en la actualidad forma parte de la zona metropolitana de la ciudad de México. El parque de Nogales sólo tiene 57 ha, divididas en 35 lotes para su renta, siguiendo la política de los parques fronterizos que alquilan tierra o naves industriales para la, algunas veces, volátil industria maquiladora. En 1986, esto es, 18 años después de su constitución, sólo renta a 16 empresas y más de la mitad de sus instalaciones no han podido ser ocupadas, por lo que se puede considerar como una modesta experiencia empresarial.

Por ser el primer parque en las inmediaciones de la ciudad de México, el Parque Industrial Cartagena es de interés particular. El 4 de diciembre 
de 1969, la sociedad denominada Parque Industrial Cartagena, S. A. de C. V., firmó un contrato de fideicomiso con una empresa bancaria, y en enero de 1970 solicitó al gobierno del estado de México la autorización para establecerse. Casi cuatro años después, el 19 de septiembre de 1973, en la Gaceta de Gobierno del estado de México se establece un acuerdo que autoriza su construcción. En general, el acuerdo señala que el fraccionamiento deberá sujetarse a las especificaciones de la Dirección de Comunicaciones y Obras Públicas, debiendo proporcionar los servicios de abastecimiento de agua, desagüe, pavimentos en las calles, banquetas en las aceras, red de distribución de energía eléctrica, camellones y alumbrado en las calles, etc. (Gobierno del Estado de México, 1973: 1).

El Parque Industrial Cartagena tiene una extensión de 63 ha divididas en 23 lotes que van de $50000 \mathrm{~m}^{2}$ hasta $4771 \mathrm{~m}^{2}$. De 1973 a 1985 , después de haber vendido todos los lotes disponibles, el fraccionador no cumplió en su totalidad con la realización de los servicios de infraestructura, faltando principalmente el alumbrado público, las guarniciones y banquetas, así como proporcionar vigilancia, recolección de basura y limpieza de calles.

Adicionalmente del incumplimiento en la dotación de infraestructura, el parque quedó sin mantenimiento alguno, situación que hizo crisis a mediados de 1985 cuando se dañó el sistema de bombeo de los pozos que lo abastecen de agua y las empresas quedaron sin el líquido aproximadamente ocho semanas. Ante la gravedad del problema, acudieron al fraccionador y éste acordó pagar $50 \%$ de la reparación del sistema hidraúlico, condicionado a que los propietarios de las empresas se hicieran cargo, en lo sucesivo, del mantenimiento. Al considerar esto inaceptable, se hicieron cargo directamente del problema teniendo que pagar alrededor de un millón de pesos para reparar el sistema de bombeo de agua.

Finalmente, de junio a diciembre de 1985, los industriales, el fraccionador y el gobierno municipal tuvieron una serie de reuniones para discutir la posibilidad de que dicho fraccionador entregara el parque al municipio. Este último, no obstante, se rehusaba a aceptar dicha entrega hasta que aquél no terminara las obras que se había comprometido a realizar. En una reunión del 17 de diciembre de 1985, el fraccionador argumentó que no contaba con recursos financieros para la construcción de la infraestructura faltante y ofreció repavimentar una calle, colocar alumbrado público y bombas para el sistema de agua. Los industriales no aceptaron el ofrecimiento y exigieron que se cumpliera con las condiciones que estipulaba la Gaceta de Gobierno del 19 de septiembre de 1973. La situación quedó en un impasse.

Esta compleja problemática del Parque Industrial Cartagena es recurrente en parques industriales privados que no cuentan con la experiencia y los recursos financieros necesarios para proyectos de muy lenta rotación que son instrumentos de políticas globales como la de 
descentralización industrial, por lo que se requiere de una optica nacional. Este caso ejemplifica que, aún desde una perspectiva microeconómica, estos proyectos necesitan de una administración permanente para su adecuado financiamiento, hecho que debe ser contemplado cuidadosamente en la concesión de permisos para su instalación.

Finalmente, en este periodo se tiene el Parque Industrial 5 de Mayo, localizado en Puebla, a $8 \mathrm{~km}$ del centro de la ciudad. Su escritura constitutiva es de 1968, cuando un grupo de empresarios inicia la construcción del Parque Industrial El Conde. Debido a problemas financieros, el gobiemo del estado continúa con su construcción y administración en 1973, cambiando el nombre a Parque Industrial 5 de Mayo. El parque es pequeño y tiene únicamente 33.2 ha de superficie total, de las cuales 29.9 son vendibles, 1.2 para servicios y 2.1 para áreas verdes. Está dividido en 35 lotes que se terminaron de vender en 1979, esto es, en un periodo de 11 años a razón de 3.2 anuales. Del total de lotes, cinco se encuentran desocupados sin construir, en dos están empresas de servicios (Depósito Conasupo y Bodegas Chopo), en otros dos lotes las plantas están cerradas (una por quiebra, otra por cambio) y existen 26 negocios manufactureros en operación. Considerando los cinco lotes baldíos, se puede decir que en 18 años se han instalado negocios a razón de 1.7 anuales, mientras que el promedio general para todos los parques y ciudades industriales considerados es de 2.8 .

Al finalizar el periodo de ventas, el gobierno del estado entrega su administración al municipio de Puebla, quien lo maneja a través de un Consejo Administrativo de Parques y Zonas Industriales (CAPZI), junto con la Asociación de Industriales, Propietarios, Inquilinos y Prestadores de Servicios del Parque Industrial 5 de Mayo de la Ciudad de Puebla.

La evolución de este proyecto que se inicia como privado y se transforma en público estatal y termina en municipal, puede considerarse sintomática del carácter "natural" de que este tipo de iniciativas sean públicas. Esto es así no sólo por la magnitud de las inversiones que reclaman y su lenta recuperación, sino porque la dotación de infraestructura y la administración permanente difícilmente puede ser rentable desde un punto de vista privado. Los parques y ciudades industriales constituyen un claro ejemplo de una de las funciones clásicas que corresponde realizar al Estado.

\section{Bibliografia}

Bale, S. R, 'Toward a Geography of Industrial Estate', en The Professional Geographer, vol. XXVI, núm. 3, agosto, 1974.

Barr, B.M, "Industrial Parks as Locational Environments: A Research Challenge", F.E.I. Hamilton, G.J.R. Linge (eds.), Spatial Analysis, Industry and the Industrial Environment vol. 3, Regional Economies and Industrial Systems, J. Wiley and Sons, 1983. 
Bharti, R.K., Industrial Estates in Developing Economies, National Publishing House, Nueva Delhi, 1978.

Bredo, W., Industrial Estates, Tool of Industrialization, International Industrial Development Center, Stanford, Research Institute, Asia Publishing House, India, 1960.

Galantay, Ervin Y., New Towns, George Braziller, Nueva York, 1975.

Garza, Gustavo, El proceso de industrialización en la ciudad de México, 1821-1970, El Colegio de México, México, 1985.

Garza, Gustavo, et al., Atlas de la ciudad de México, Departamento del Distrito Federal, El Colegio de México, México, 1987.

La Jornada, 24 de octubre de 1987.

Miller, Frank C., Old Village and a New Town: Industrialization in Mexico, Cummings Publishing Company, Menlo Park, 1973.

Naciones Unidas, Industrial Estates in Europe and The Middle East, United Nations Industrial Development Organization, Nueva York, 1968.

Porter, B.M., A Model Industrial Park Worland, Wgo, The Library of the University of California, Los Angeles, $s / f$.

Unikel, L., C. Ruiz y G. Garza, El desarrollo urbano de México, El Colegio de México, México, 1976.

, El desarrollo urbano de México, El Colegio de México, México, 1976. 